\title{
Réflexion autour de la fonction des retouchoirs élise tartar en os de l'Aurignacien ancien
}

\begin{abstract}
Résumé
Les retouchoirs en os représentent le plus ancien outillage en matières dures animales $d u$ Paléolithique européen. Ils se rencontrent très fréquemment au sein des ensembles moustériens et perdurent tout au long du Paléolithique supérieur. Plusieurs études fonctionnelles leur ont été consacrées et l'hypothèse la plus communément admise est celle de leur emploi dans la retouche de tranchants lithiques. Cette hypothèse repose toutefois principalement sur l'étude de pièces moustériennes alors que les exemplaires du Paléolithique supérieur, en particulier ceux de l'Aurignacien ancien, présentent des caractéristiques légèrement différentes. À partir de l'étude de plusieurs séries de l'Aurignacien ancien (l'abri Castanet en Dordogne, la grotte des Hyènes dans les Landes et la grotte Gatzarria dans les Pyrénées-Atlantiques) et d'une reconstitution expérimentale, nous montrons que les retouchoirs de cette période témoignent d'une évolution de leur mode de fonctionnement, liée aux changements opérés dans le domaine lithique. Nous envisageons également la possibilité de leur emploi dans le débitage lamellaire.
\end{abstract}

Mots-clés

Aurignacien ancien, retouchoirs en os, technologie osseuse, approche fonctionnelle, expérimentation, retouche laminaire, débitage lamellaire.

\section{Abstract}

Bone retouchers are the most ancient osseous tools of the European Palaeolithic. They are very frequent on Mousterian sites and persist throughout the Upper Palaeolithic. Several functional analyses have been devoted to them and the most commonly accepted hypothesis proposed is their use for lithic tool retouching by percussion. However, this hypothesis is based mainly on studies of Mousterian pieces while few functional analyses have been undertaken on Upper Palaeolithic bone retouchers. The persistent presence of these "ad hoc" tools makes their study important.

This article presents the results of a techno-functional analysis devoted to the Early Aurignacian bone retouchers from Abri Castanet at Sergeac (Dordogne), northern and southern sectors (layer A and US 131 and below), Grotte des Hyènes at Brassempouy (Landes, complex 2) and Gatzarria at Suhare (Pyrénées-Atlantiques, layer cbcf). These unworked bone tools, including many specimens identified a posteriori among faunal remains, represent a significant part of the studied assemblages' osseous equipment. There are two categories of retouchers: those on raw bone fragments and those on technical objects. The former, most numerous, are mainly long bone shaft fragments recovered after food operations and used just as they were, without modification. The latter correspond to the use of finished 
objects like smoothers and awls as well as manufacturing by-products. They bear diagnostic traces of utilisation in the form of linear, deep, short depressions, $v$-shaped in cross section. These depressions are similar to those that have been described in previous work involving microscopic observation and are compatible with use for lithic tool retouching by percussion. However, unlike Middle Palaeolithic retouchers, where the depressions are perpendicular to the main axis of the piece, Early Aurignacian depressions are often vertical (in combination or not with horizontal ones). This is a characteristic feature of Upper Palaeolithic retouchers related to their positions during use. The vertical depressions imply that the main axis of the retoucher is oriented parallel to the lithic edge during use. Quite obviously, this change in the functioning of retouchers must be linked to changes in flint-knapping processes. To understand this relationship, we explored the effects of changing the orientation of retouchers. One notable difference is their curvature in the transverse and longitudinal axes. The latter is clearly more pronounced than the former, meaning that the retouchers offer a greater overall surface area when used in a parallel orientation.

We hypothesized that this orientation was preferred to increase the active surface of retouchers for the retouching of blade products. Indeed, during the Early Aurignacian, the manufacture of blade tools, scrapers in particular, often involved retouching very convex and narrow ends. Orienting the retoucher parallel to the lithic edge would have been a technical solution to compensate for the decrease in the active surface presented by the strong convexity of blade ends. However, within the Early Aurignacian lithic industry, blade ends are not the only items to show strong convexity: the fronts of carinated and nosed scrapers also display them. The use of retouchers in bladelet knapping has not so far been considered, but constitutes a second hypothesis that could explain the particular orientation of Early Aurignacian retouchers during use.

These two assumptions have been tested by experimental replication. Results show that bone retouchers are effective tools for both blade retouching and bladelet knapping, provided that the weight of the retoucher is adjusted to suit the type of lithic product. In addition, lithic and bone tools produced experimentally fall within the range of variation observed in the archaeological assemblages studied. Finally, although this has not always been necessary, experimentation confirms the usefulness of parallel retoucher orientation to adapt the active surface areas to strong lithic convexity. To date, strong convexity of lithic products is the main factor likely to explain the change in retoucher orientation that occurred at least as early as the Early Aurignacian. Thus, retouchers of this period attest to the duration of an ancient technical process, involving the use of bone for stone knapping. Specimens bearing vertical depressions thus illustrate the adaptation of the retouchers to "new" lithic technologies.

The important role played by retouchers in retouching Mousterian tools and their number within the assemblages studied leave little doubt as to their use in retouching Upper Palaeolithic blade products. However, it is still difficult to determine whether they were in fact used in bladelet knapping. The traces of utilisation on experimental retouchers are quite similar to those on specimens used in blade retouching. An expanded experimental program and analysis of the bone tools with high magnification should facilitate the assessment of morphometric changes in use-wear traces based on activity. In addition, a comparison of technical and functional characteristics of archaeological and experimental lithic products should help clarify the array of hammers used during the Aurignacian.

Keywords

Early Aurignacian, bone retouchers, osseous technology, functional approach, experimentation, blade retouching, bladelet knapping. 


\section{INTRODUCTION}

Les retouchoirs en os constituent sans conteste le plus ancien outillage en matières dures animales du Paléolithique européen. Ils seraient en effet attestés dès le Paléolithique inférieur, se rencontrent très fréquemment au sein des ensembles du Paléolithique moyen et perdurent tout au long du Paléolithique supérieur. Témoins tangibles d'une exploitation précoce de l'os à des fins techniques, ils sont identifiés dès la fin du $\mathrm{XIX}^{\mathrm{e}}$ siècle (Leguay, 1877; Daleau, 1883). L. Henri-Martin est le premier à en faire une étude approfondie à partir des exemplaires des niveaux moustériens de La Quina (1907-1910). Depuis, plusieurs travaux incluant des reconstitutions expérimentales ont porté sur cet outillage, et si différentes fonctions ont été proposées (retouchoirs, compresseurs, enclumes, poussoirs à aiguille, etc.), l'hypothèse aujourd'hui la plus communément admise est celle d'un emploi dans la retouche de tranchants lithiques (notamment Chase, 1990; Vincent, 1993; Armand et Delagnes, 1998; Karavanic et Sokec, 2003). Cependant, peu d'études fonctionnelles impliquant des reconstitutions expérimentales ont encore été entreprises sur les retouchoirs en os du Paléolithique supérieur. La permanence de ces outils qualifiés «de fortune» mérite pourtant que l'on s'y intéresse. Font exception les travaux conduits par A. Rigaud et C. Schwab (Rigaud, 1977 et 2007; Schwab, 2009). En 1977, A. Rigaud proposait un emploi dans l'affûtage de grattoirs pour les retouchoirs magdaléniens de La Garenne à Saint-Marcel (Indre), une hypothèse qu'il réitéra en 2007 pour les exemplaires de Labastide (Hautes-Pyrénées). En 2009, C. Schwab, en collaboration avec A. Rigaud, publia les résultats d'une étude diachronique des retouchoirs d'Isturitz provenant des niveaux moustériens, aurignaciens, gravettiens et magdaléniens. Constatant une évolution chronologique au niveau des stigmates d'utilisation des retouchoirs, l'auteur proposa que ces changements reflètent les différents «modes de retouche» réalisés sur l'équipement lithique (Schwab, 2009, p. 32).

Cet article présente les résultats d'une étude technofonctionnelle consacrée aux retouchoirs de l'Aurignacien ancien (Tartar, 2009 et à paraître). À partir des exemplaires de l'abri Castanet (Sergeac, Dordogne), de la grotte des Hyènes (Brassempouy, Landes) et de Gatzarria (Suhare, Pyrénées-Atlantiques), nous montrons que cet outillage peu élaboré représente une part importante, voire majoritaire, de l'équipement en matières osseuses. Notre propos se concentre ensuite sur les résultats de l'analyse fonctionnelle, menée avec l'appui de l'expérimentation. Nous démontrons que ces retouchoirs de l'Aurignacien ancien, tout en conservant des caractéristiques très proches de celles des exemplaires moustériens, ont fait l'objet d'un mode de fonctionnement différent lié aux changements opérés dans le domaine lithique. Notre étude menée en parallèle des travaux récents de A. Rigaud et C. Schwab (Rigaud, 2007 et Schwab, 2009), vient ainsi appuyer les données de ces auteurs. Toutefois, nous envisageons également la possibilité que l'emploi des retouchoirs ne se soit pas limité à des activités de retouche.

\section{RAPPEL DES CARACTÉRISTIQUES FONCTIONNELLES ET DU FONCTIONNEMENT DES RETOUCHOIRS}

Les retouchoirs se caractérisent par des plages d'impressions localisées à proximité des extrémités (fig. 1). Les études ayant impliqué une observation microscopique de ces impressions en donnent des descriptions très semblables et cela, indépendamment semble-t-il, du type de supports exploités et de leur contexte de découverte (notamment Vincent, 1993; Malerba et Giacobini, 1996; Patou-Mathis, 2002; Tartar, 2009).

Ces impressions correspondent à l'empreinte laissée par un tranchant lithique (fig. 1). Il n'est pas rare, d'ailleurs, d'observer des micro-esquilles de silex encore fichées dans la matière, au fond de ces stigmates. Une impression est une dépression à section en «V» asymétrique. Elle est donc formée par deux pans d'aspect distinct qui permettent de reconstituer le fonctionnement de l'outil (Malerba et Giacobini, 1996; Tartar, 2009). Le pan abrupt correspond à la pénétration, plus ou moins oblique, du tranchant lithique dans la matière osseuse. Sous la force exercée, la matière se compacte et se couvre de stries microscopiques liées aux microirrégularités du tranchant. Le pan d'arrachement, plus irrégulier, correspond à sa sortie et s'apparente souvent à un négatif d'enlèvement de copeau (fig. 1). Cette cinématique de l'outil est confirmée par la présence, presque systématique, de fines stries partant du pan d'arrachement des impressions : elles correspondent au glissement du retouchoir sur le tranchant lithique.

Ces observations évoquent un fonctionnement en percussion et les études ayant engagé des reconstitutions expérimentales s'accordent pour un emploi dans la retouche de tranchants lithiques, pour la confection et l'entretien des outils (voir notamment Chase, 1990).

\section{LES RETOUCHOIRS EN OS DE L'AURIGNACIEN ANCIEN}

\section{Le corpus d'étude}

Notre étude a porté sur les retouchoirs aurignaciens anciens de trois gisements : l'abri Castanet (Sergeac, Dordogne), la grotte des Hyènes (Brassempouy, Landes) et la grotte Gatzarria (Suhare, PyrénéesAtlantiques). Les retouchoirs de l'abri Castanet se répartissent en deux séries : l'une provenant du secteur nord (couche A, fouilles de D. Peyrony: Peyrony, $1935)^{1}$ et l'autre du secteur sud (US 131 et sousjacentes) fouillé entre 1995 et 1998 (fouilles de J. Pelegrin et R. White) et entre 2005 à 2010 (fouilles de R. White $)^{2}$. Elles totalisent respectivement 78 et 88 retouchoirs $^{3}$. Les exemplaires de la grotte des Hyènes proviennent de l'ensemble 2 auxquels nous avons ajouté les pièces issues des unités stratigraphiques I1 et I2 de l'abri voisin, l'abri Dubalen (fouilles de H. Delporte, D. Buisson, D. Henry-Gambier et F. Bon, entre 1981 et 2002). Ces unités stratigraphiques 


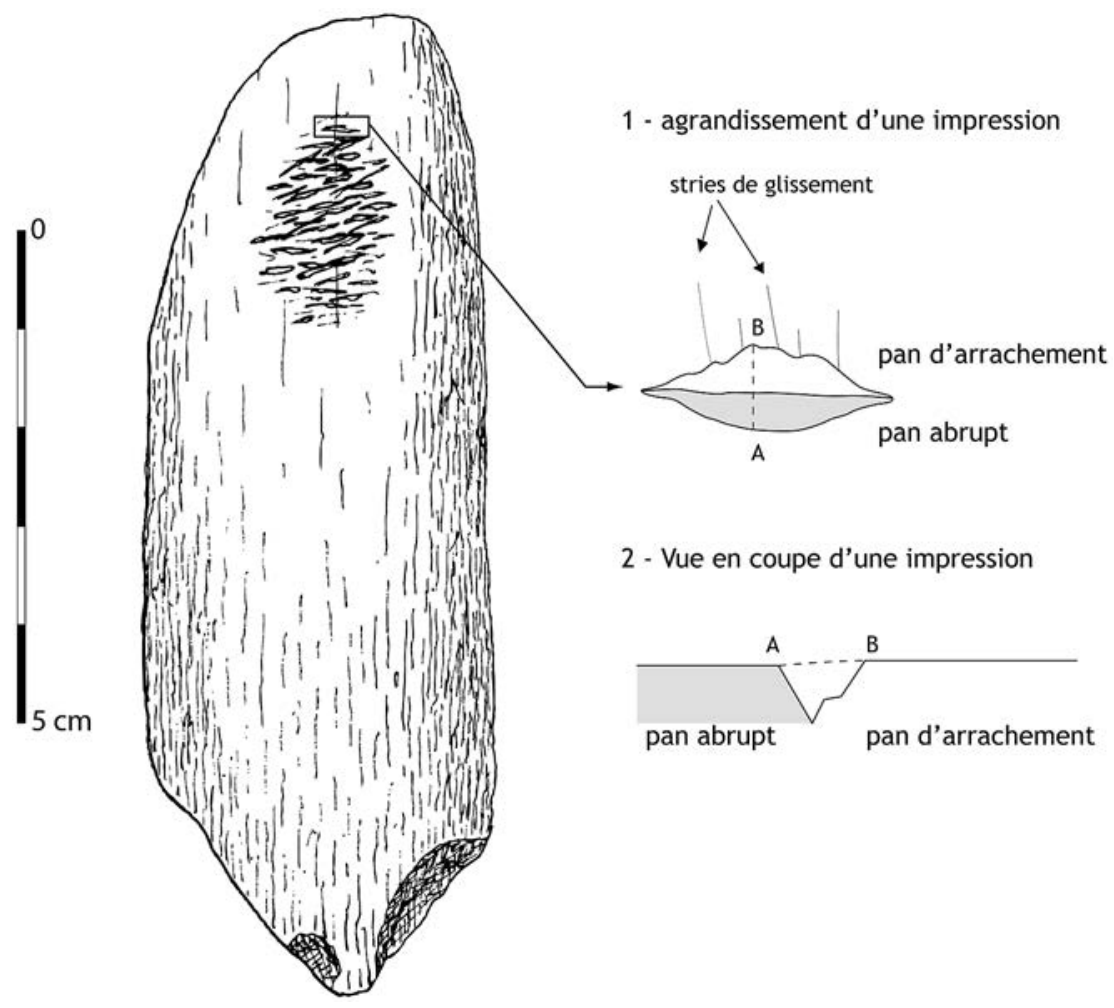

Fig. 1 - Morphologie des impressions (dessin : F. Le Mené, DAO : F. Tessier).

Fig. 1-Morphology of use-wear marks (drawing: Florent Le Mené, CAD: F. Tessier).

sont en effet en position secondaire et proviennent d'un glissement de dépôts aurignaciens anciens de la grotte des Hyènes (Henry-Gambier et al., 2004) ${ }^{4}$. L'ensemble représente 87 pièces. Enfin, les retouchoirs de Gatzarria sont au nombre de 92 et proviennent de la couche cbcicbf (fouilles de G. Laplace de 1961 à 1976 : Laplace, 1966).

\section{La place des retouchoirs au sein de l'industrie osseuse}

Les recherches menées jusqu'à présent sur les retouchoirs se sont concentrées essentiellement sur des séries du Paléolithique moyen (Henri-Martin, 19071910; Chase, 1990; Vincent, 1993; Armand et Delagnes, 1998; Karavanic et Sokec, 2003). Ces outils représentent pourtant un composant classique de l'équipement osseux de fonds commun du Paléolithique supérieur, à l'Aurignacien ancien notamment (fig. 2).

De fait, si on fait exception du secteur nord de l'abri Castanet - le matériel a fait l'objet d'une récolte sélective lors des fouilles de D. Peyrony et l'effectif des outils peu transformés, notamment les retouchoirs, est nettement sous-évalué - ils constituent au moins la moitié de l'équipement en os mis au jour (50\% à la grotte des Hyènes, 57 \% à Gatzarria). Ils représentent même l'essentiel de l'outillage en os récolté dans le secteur sud de l'abri Castanet (94\% de l'outillage). La faible étendue des fouilles et l'échantillonnage du matériel étudié nécessitent toutefois de pondérer ces données.

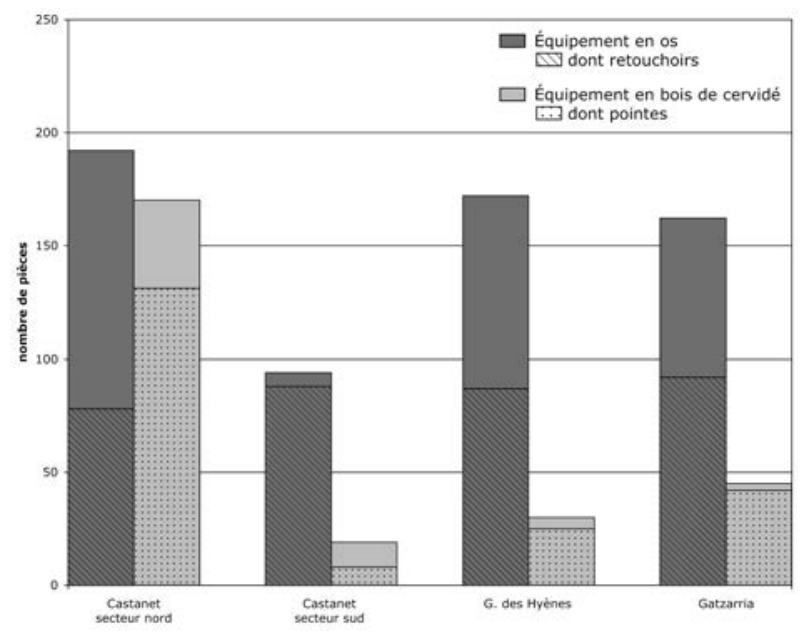

Fig. 2 - Composition de l'équipement en matières osseuses de l'abri Castanet secteur nord (couche A) et secteur sud (US 131 et sous-jacentes), grotte des Hyènes (ensemble 2) et Gatzarria (couche cbci-cbf).

Fig. 2-Osseous tools in Abri Castanet northern sector (layer A) and southern sector (US 131 and below), Grotte des Hyènes (complex 2) and Gatzarria (layer cbci-cbf).

Plus marquant encore est sans doute le fait que, sauf pour le secteur nord de l'abri Castanet, le nombre de retouchoirs est largement supérieur à celui des pointes en bois de cervidé, et même à l'équipement en bois de cervidé pris dans son ensemble (fig. 2). À cet égard, les recherches menées jusqu'à présent sur l'industrie en matières osseuses aurignacienne ont surtout mis 
l'accent sur le bois de cervidé, notamment sur les pointes, entretenant ainsi l'idée d'un outillage en os peu abondant. Concernant plus spécifiquement les retouchoirs, le problème vient aussi d'un défaut de reconnaissance. Même lors des fouilles les plus récentes, ces pièces ne sont jamais toutes identifiées et une part conséquente (entre un tiers et plus de la moitié selon les cas) doit être récupérée postérieurement au sein des restes de faune.

Les retouchoirs de l'Aurignacien ancien témoignent donc de la persistance d'un procédé technique moustérien impliquant l'exploitation de l'os dans la transformation de la matière lithique et montrent que ce procédé est encore fortement prégnant. Les caractéristiques des outils osseux sont cependant différentes, ce qui est perceptible notamment dans le choix des supports exploités.

\section{Les supports exploités}

Deux types de supports ont été exploités : des supports bruts et des pièces techniques (tabl. 1).

Les retouchoirs sur supports bruts - ou plus simplement les «retouchoirs bruts»-, les plus nombreux, sont très semblables à ceux des ensembles du Paléolithique moyen. Ce sont principalement des fragments d'os, vraisemblablement récupérés à l'issue des opérations alimentaires et utilisés tels quels (fig. $3, \mathrm{n}^{\mathrm{o}} 1$ ). En effet, l'hypothèse d'une production intentionnelle de supports apparaît improbable. Dans les trois sites étudiés, les os longs ont été systématiquement fracturés dans le but de recueillir la moelle. Or, nous n'avons pas constaté de différences morphométriques notables entre les outils et les fragments d'os, présents dans la faune et non utilisés. Les retouchoirs bruts comprennent également des exemplaires sur dents, plus rares. Ils sont connus dès le Moustérien (couche 6c d'Artenac: Armand et Delagnes, 1998) mais il s'agit alors essentiellement de dents jugales de cheval (présents aussi dans l'Aurignacien ancien de La Ferrassie : Castel et al., 2003). Des retouchoirs sur canines de grands carnivores ont, en revanche, été identifiés dans plusieurs gisements, au sein de dépôts attribués à l'Aurignacien ancien (La Ferrassie, La Souquette : Leroy-Prost, 2002; Castel et Madeleine, 2003; Le Piage : Tartar, 2006; etc.). La couche $2 \mathrm{Cc}$ de la grotte des Hyènes en a livré trois exemplaires, sur canines d'ours (fig. 3, no 2). Contrairement à ce qui a pu être proposé (notamment Leroy-Prost, 1975, p. 145; Castel et al., 2003, p. 34), ils n'ont toutefois pas valeur de marqueur culturel pour la phase ancienne, dans la mesure où plusieurs exemplaires sont associés à d'autres phases de l'Aurignacien ${ }^{5}$.

Les retouchoirs sur pièces techniques ne se rencontrent que dans les ensembles du Paléolithique supérieur, mais sont rarement signalés dans les études et publications (sauf, par exemple, Didon, 1911; Vézian et Vézian, 1970; Schwab, 2009). Ce sont des outils de seconde intention, correspondant à l'emploi secondaire d'objets finis (façonnés) ou de restes de fabrication (fig. 3, $\mathrm{n}^{\circ} 3$ ). Pour nos séries, Il s'agit d'objets sur côtes, surtout des lissoirs, ainsi que des poinçons sur os longs. L'absence fréquente de recouvrement entre les deux registres de stigmates d'usure et l'état de conservation des pièces rendent difficile la reconstitution de la chronologie des emplois. Quand cela a été possible, nous avons pu constater que ces retouchoirs de seconde intention relevaient souvent d'un recyclage - l'outil premier a été définitivement détourné de sa fonction initiale - mais dans certains cas également d'un emploi secondaire ponctuel l'outil premier a continué à remplir sa fonction initiale.

L'exploitation d'une plus large gamme de produits (canine de grands carnivores, pièces techniques) est donc un élément important distinguant les retouchoirs de l'Aurignacien ancien et les exemplaires du Paléolithique moyen étudiés jusqu'à présent. À l'exception peut-être des canines de grands carnivores, cette plus large exploitation tient vraisemblablement au plus grand éventail de supports disponibles. Toujours est-il que du point de vue fonctionnel, la différence la plus marquante entre les retouchoirs de l'Aurignacien ancien et du Paléolithique moyen concerne l'orientation des stigmates d'usure.

\begin{tabular}{|c|c|c|c|c|c|}
\hline & \multirow{2}{*}{\multicolumn{2}{|c|}{ Castanet }} & \multirow{3}{*}{ Brassempouy } & \multirow{3}{*}{ Gatzarria } & \multirow{3}{*}{ Total } \\
\hline & & & & & \\
\hline & s. nord & s. sud & & & \\
\hline Retouchoirs bruts & 54 & 83 & 71 & 85 & 297 \\
\hline sur os longs & 54 & 83 & 68 & 85 & 294 \\
\hline sur dents & - & - & 3 & - & 3 \\
\hline Retouchoirs sur pièces techniques & 24 & 5 & 16 & 7 & 52 \\
\hline sur lissoirs & 18 & 1 & 10 & 5 & 34 \\
\hline émi-côtes en cours de transformation & 2 & 2 & 2 & 1 & 7 \\
\hline sur côtes décorées & 1 & - & & 1 & 2 \\
\hline sur outil intermédiaire & - & - & 1 & - & 1 \\
\hline sur poinçons & 3 & 2 & 3 & - & 8 \\
\hline Total & 78 & 92 & 87 & 92 & 349 \\
\hline
\end{tabular}

Tabl. 1 - Inventaire des retouchoirs en os de l'abri Castanet secteur nord (couche A) et secteur sud (US 131 et sous-jacentes), grotte des Hyènes (ensemble 2) et Gatzarria (couche cbci-cbf).

Table 1 - Inventory of bone retouchers at Abri Castanet northern sector (layer A) and southern sector (US 131 and below), Grotte des Hyènes (complex 2) and Gatzarria (layer cbci-cbf). 

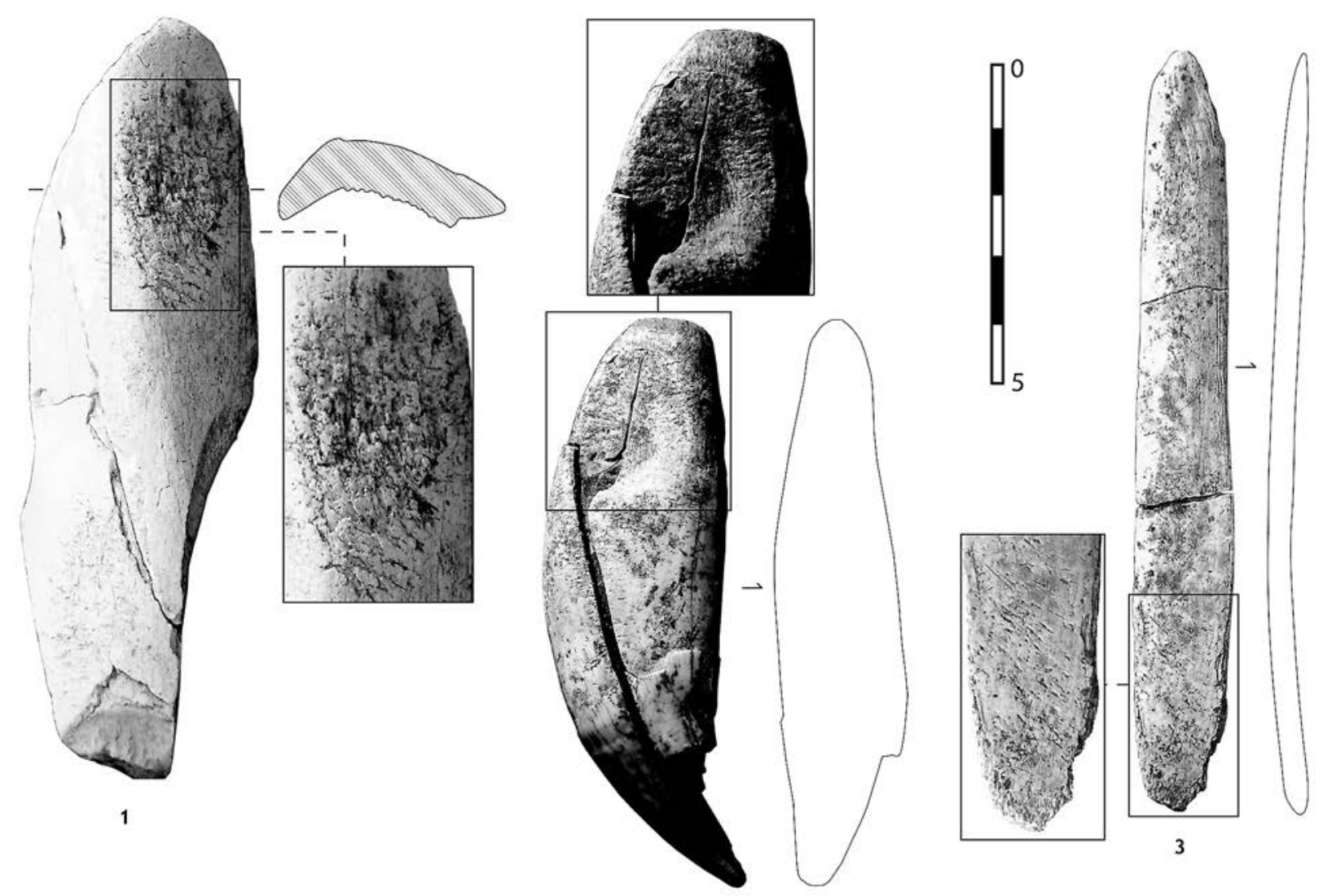

Fig. 3 - Exemples de retouchoirs bruts et sur pièces techniques, 1 : retouchoir brut sur fragment diaphysaire portant des impressions horizontales et verticales (Castanet, secteur nord, couche A) ; 2 : retouchoir brut sur canine d'ours (BD4-2051, grotte des Hyènes, 2Cc); $3:$ retouchoir sur lissoir (BA12-1076, grotte des Hyènes, 2E).

Fig. 3 - Examples of unworked retouchers and retouchers on manufactured pieces, 1: unworked retoucher on fragment of long bone shaft with horizontal and vertical use-wear marks (Castanet, northern sector, layer A); 2: unworked retoucher on bear canine (BD4-2051, Grotte des Hyènes, $2 C c)$; 3: retoucher on smoother (BA12-1076, Grotte des Hyènes, 2E).

\section{L'orientation des stigmates d'utilisation}

Les études menées sur les retouchoirs du Paléolithique moyen décrivent des impressions horizontales ou légèrement obliques (notamment Henri-Martin, 1907-1910; Vincent, 1993; Armand et Delagnes, 1998; et obs. pers.). En revanche, les retouchoirs de nos séries (bruts et sur pièces techniques) présentent fréquemment des impressions verticales (ou légèrement obliques) ${ }^{6}$ : fig. $3, \mathrm{n}^{\circ} 1$. Ainsi, les plages d'utilisation comportant des impressions verticales (uniquement ou non) représentent, selon les séries, entre 33 et $68 \%$ des plages (entières) des retouchoirs (33\% pour Castanet-sud, soit $\mathrm{n}=19 ; 34 \%$ pour Gatzarria, soit $\mathrm{n}=21 ; 63 \%$ pour La grotte des Hyènes, soit $n=37 ; 68 \%$ pour Castanetnord, soit $n=40)$ : fig. 4 .

L'orientation des impressions est liée au positionnement de l'outil lors de l'utilisation (fig. 5). Les impressions horizontales supposent une orientation perpendiculaire du retouchoir, autrement dit que son axe d'allongement est placé perpendiculairement au tranchant lithique lors de l'utilisation tandis que les impressions verticales impliquent une orientation parallèle. Cette orientation différentielle des stigmates - et ce faisant celle de l'outil - est loin d'être anodine puisque les impressions verticales sont une caractéristique propre aux retouchoirs du Paléolithique supérieur (Giraux, 1907; Rigaud, 2007; Schwab, 2002 et 2009). Selon tout évidence et comme l'a proposé C. Schwab (op. cit.), cette modification du fonctionnement des outils doit être rapprochée de changements opérés dans le domaine lithique.

Pour comprendre cette relation, nous nous sommes demandé quel intérêt il y aurait à modifier l'orientation des retouchoirs. Une différence notable réside dans leur courbure selon l'axe transversal et longitudinal : cette dernière est nettement plus marquée dans le premier cas que dans le second (pour les retouchoirs sur fragments d'os longs, compte tenu de la forme cylindrique des diaphyses, mais aussi pour les outils sur pièces techniques, qu'il s'agisse de lissoirs, de poinçons, etc.). À cet égard, si on étudie la courbure des retouchoirs au niveau des plages d'utilisation associant des impressions horizontales et verticales (fig. 6), on constate qu'hormis quelques plages occupant une surface de courbure identique dans les deux axes, les impressions se présentent sur des zones plus convexes dans l'axe transversal que dans l'axe longitudinal. Cela implique que les retouchoirs offrent globalement une plus grande surface active lorsqu'ils sont utilisés selon 


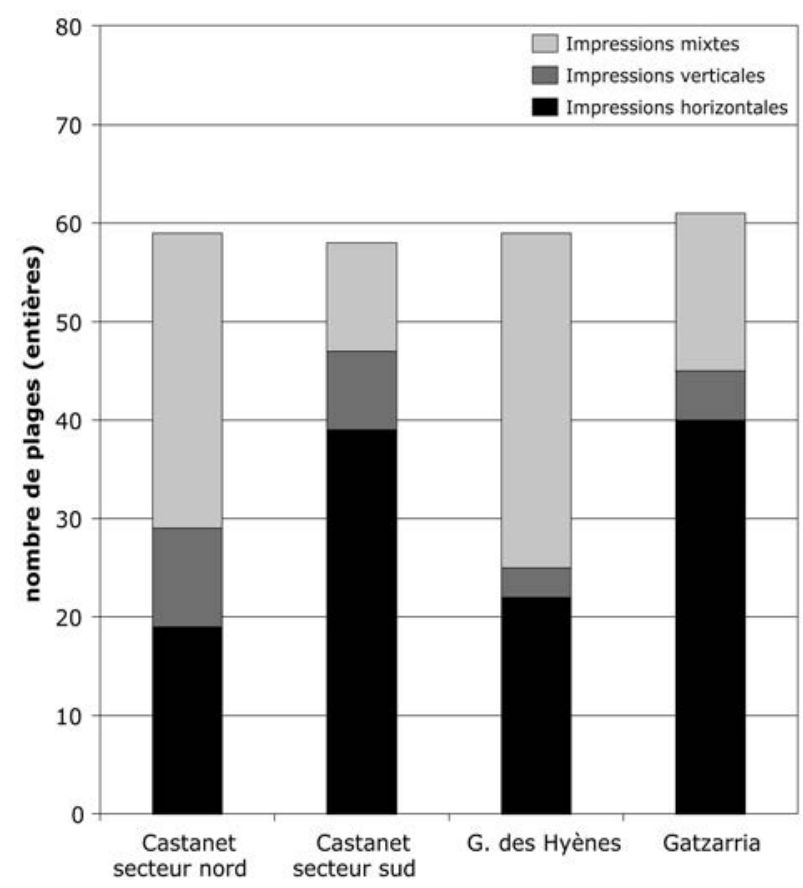

Fig. 4 - Orientation des impressions au sein des plages des retouchoirs de l'abri Castanet secteur nord (couche A) et secteur sud (US 131 et sous-jacentes), grotte des Hyènes (ensemble 2) et Gatzarria (couche cbci-cbf). Seules les plages entières ont été prises en compte ce qui explique les écarts d'effectif avec le corpus total étudié.

Fig. 4 - Orientation of use wear marks on retouchers from Abri Castanet northern sector (layer A) and southern sector (US 131 and below), Grotte des Hyènes (complex 2) and Gatzarria (layer cbci-cbf). Only full use-wear areas have been taken into account. une orientation parallèle. Aussi avons-nous émis l'hypothèse que cette orientation a été privilégiée pour augmenter la surface active des retouchoirs, et cela dans le cadre de la retouche des produits laminaires. En effet, la confection et l'entretien de l'outillage sur lame ont souvent impliqué d'en retoucher les extrémités, notamment dans le cas des grattoirs. Or ces zones étroites présentent des délinéations très convexes ce qui réduit sensiblement la portion du tranchant en contact avec le retouchoir. Autrement dit, orienter le retouchoir parallèlement au tranchant a pu constituer une solution technique pour compenser la diminution de surface active induite par la forte convexité des extrémités de lames. Cette hypothèse pourrait également expliquer le fait que les retouchoirs à impressions verticales ne se rencontrent pas dans les ensembles du Paléolithique moyen compte tenu de l'absence d'un outillage standardisé sur supports laminaires à cette période. Notons que cette hypothèse rejoint celle émise par C. Schwab qui suppose que l'adoption d'une orientation parallèle pour les retouchoirs est liée à l'aménagement de front de grattoir sur lame (Schwab, 2009).

Toutefois, l'utilisation des retouchoirs ne s'est peutêtre pas limitée à des activités de retouche. En effet, au sein de l'industrie lithique de l'Aurignacien ancien, les extrémités de lames ne sont pas les seules à afficher une forte convexité. C'est le cas également du front des grattoirs carénés et à museau. Les recherches actuelles sont unanimes pour leur accorder le statut de
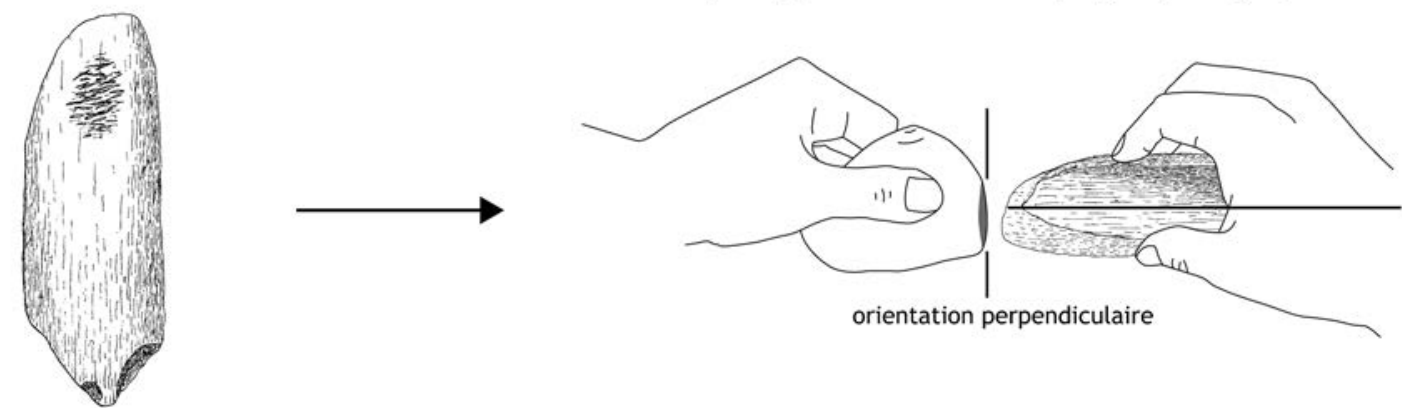

impressions horizontales

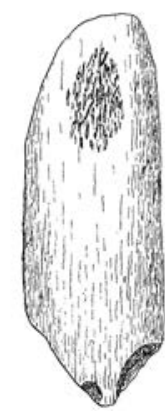

impressions verticales

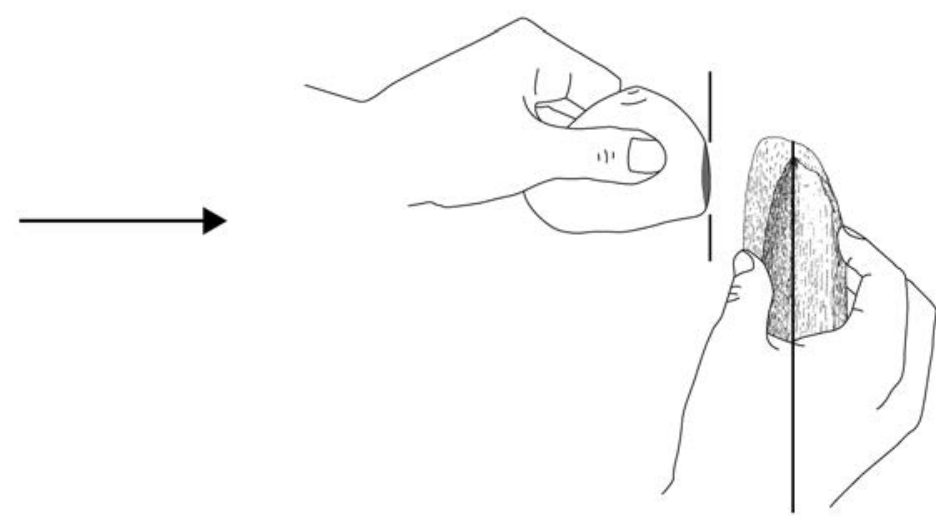

orientation parallèle

Fig. 5 - Orientation des impressions et des retouchoirs lors de l'utilisation (dessin : F. Le Mené, DAO : F. Tessier). Fig. 5 - Orientation of use-wear marks and retoucher during use (drawing: F. Le Mené, CAD: F. Tessier). 


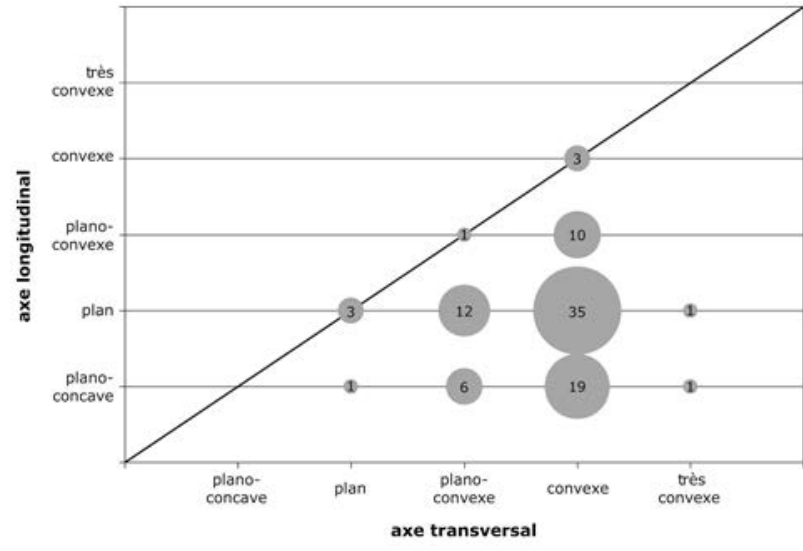

Fig. 6 - Courbure transversale et longitudinale des retouchoirs au niveau des plages d'impressions associant des impressions horizontales et verticales. Les exemplaires de Castanet secteur nord (couche A) et secteur sud (US 131 et sous-jacentes), grotte des Hyènes (ensemble 2) et Gatzarria (couche cbci-cbf) sont considérés ensemble.

Fig. 6-Transverse and longitudinal curve of retouchers in use areas combining horizontal and vertical use-wear marks. Retouchers from Abri Castanet northern sector (layer A) and southern sector (US 131 and below), Grotte des Hyènes (ensemble 2) and Gatzarria (layer cbci-cbf) are considered together.

nucléus à lamelles (voir notamment Hahn, 1970; Chiotti, 2000 pour la démonstration par le remontage, et Le Brun-Ricalens, 2005 pour une synthèse) et rapportent l'emploi de la percussion tendre. Encore jamais envisagée, la participation des retouchoirs au débitage des lamelles, en complément des percuteurs tendres classiques, constitue donc une seconde hypothèse susceptible d'expliquer l'orientation particulière donnée aux retouchoirs de l'Aurignacien ancien lors de l'utilisation.

\section{EXPÉRIMENTATION}

\section{Objectifs et protocole}

Nos expériences, réalisées avec la collaboration de deux tailleurs expérimentés, Michel Brenet et Florent Le Mené, ont consisté à utiliser huit fragments d'os (sélectionnés parmi une vingtaine) pour confectionner une dizaine de grattoirs sur lame et lames aurignaciennes ainsi que pour débiter quatre nucléus carénés et à museau. Les fragments sélectionnés proviennent d'os frais de bœuf et ont été obtenus selon les mêmes modalités que les pièces archéologiques : six fragments diaphysaires par l'éclatement d'os longs et deux hémicôtes par bipartition. Nous avons cherché à répondre à un double objectif :

- évaluer l'efficacité des retouchoirs dans les deux registres d'activités (retouche et débitage);

- vérifier le bien-fondé de la relation établie entre l'orientation parallèle des retouchoirs et la taille de tranchants lithiques à forte délinéation convexe.

Les tailleurs ont travaillé séparément, ce qui s'est révélé profitable, les séries de tests ayant abouti à des résultats comparables ${ }^{7}$. Nous avons accordé une attention particulière aux stratégies adoptées par les tailleurs. Laissés libres de leurs choix, ils ont commenté et justifié leurs décisions dans le cadre des différentes activités - en particulier concernant la sélection des fragments d'os et leur orientation par rapport au tranchant lithique. Nous leur avons toutefois fréquemment demandé de procéder différemment, voire à l'opposé de leur choix, de façon à percevoir clairement les conséquences sur le fonctionnement des outils.

\section{Les données expérimentales}

Avant que nous les sollicitions, les deux tailleurs n'avaient jamais utilisé de retouchoirs en os et doutaient de leur efficacité. Ils ont cependant rapidement constaté que les fragments d'os, bien choisis, peuvent constituer des outils de taille performants. Selon eux, rien ne vaut un bon percuteur en bois de cervidé. Cependant, si les retouchoirs nécessitent d'appuyer un peu plus les coups, ils ont l'avantage d'être moins violents à l'impact ce qui limite les risques de fracture des supports lithiques. En outre, comme le souligne A. Rigaud, les retouchoirs sur fragments diaphysaires sont «des percuteurs denses mais légers, d'une dureté légèrement supérieure à celle du bois végétal et animal et inférieure à celle de la pierre tendre, dureté qui s'accentue rapidement avec la dessiccation » (Rigaud, 2007, p. 194).

\section{La retouche de lames : confection de grattoirs et de lames aurignaciennes.}

Les fragments diaphysaires de bœuf ainsi que les hémi-côtes ont permis, avec une certaine efficacité, d'aménager des lames d'épaisseurs variables (comprise entre 9 et $19 \mathrm{~mm}$ ), par une retouche variable elle aussi (abrupte à envahissante selon l'épaisseur du support retouché). Les grattoirs et les lames aurignaciennes réalisés offrent des caractéristiques proches des exemplaires rencontrés en contexte archéologique (fig. 7, $\mathrm{n}^{\text {os }} 2$ et 5).

Pour retoucher les bords de lame, les tailleurs ont spontanément adopté une orientation perpendiculaire pour le retouchoir de façon à profiter de la convexité transversale de l'outil. Comme l'a également expérimenté A. Rigaud (2007, p. 194), l'orientation parallèle est d'ailleurs à proscrire : la surface plane de l'outil osseux, associée à la rectitude des bords de lame, multiplie les points de contact entre les deux matières et interdit toute tentative (fig. 8).

Pour retoucher l'extrémité des supports lithiques, l'orientation parallèle du retouchoir est en revanche très efficace. Comme nous l'avions envisagé, la plus grande rectitude du retouchoir dans l'axe longitudinal permet de compenser la forte convexité de l'extrémité de la lame et de conserver ainsi une surface active suffisante. Cela étant, un changement d'orientation n'est pas toujours nécessaire (fig. 8). Tout dépend en fait du degré de convexité de la surface du retouchoir 

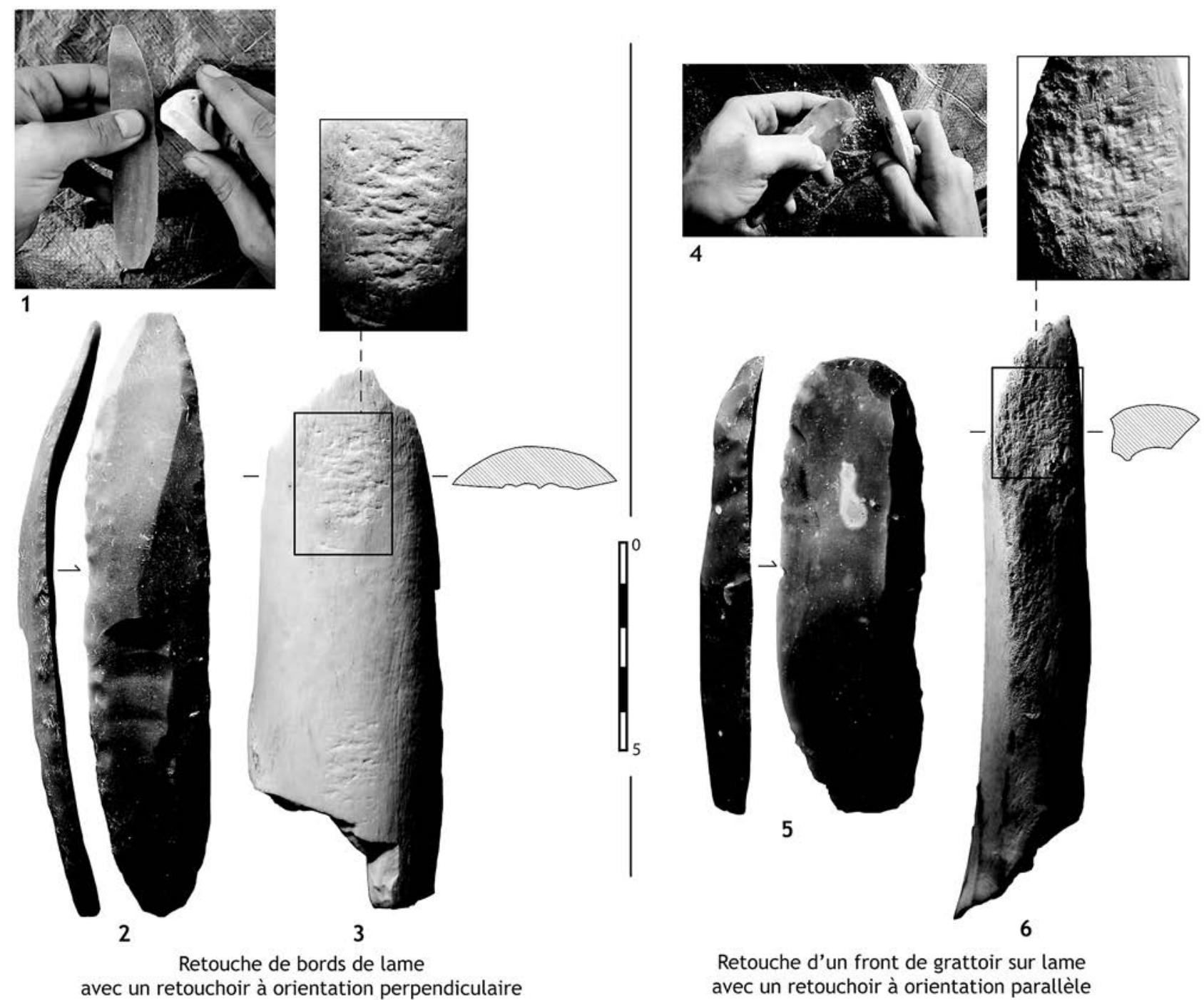

4
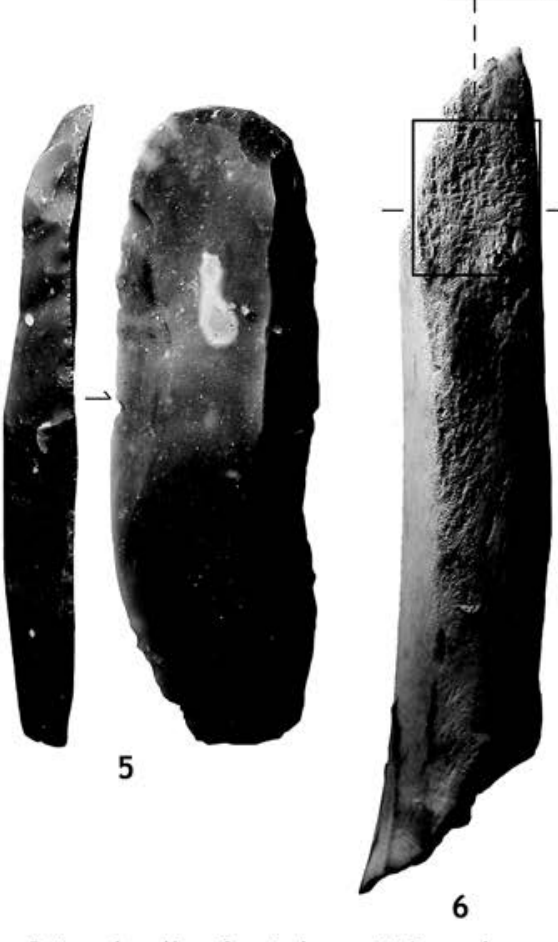

Retouche d'un front de grattoir sur lame avec un retouchoir à orientation parallèle

Fig. 7 - Utilisation expérimentale des retouchoirs pour la retouche de produits laminaires. Retouche de bords de lame, $1:$ retouchoir orienté perpendiculairement au bord; 2 : lame retouchée; 3 : retouchoir avec impressions horizontales. Retouche de front de grattoir sur lame, 4 : retouchoir orienté parallèlement au front 5 : grattoir sur lame; 6 : retouchoir avec impressions verticales.

Fig. 7 - Experimental use of retouchers on blades. Retouches on blade edges, 1: retoucher oriented perpendicular to the edge; 2 : retouched blade; 3: retoucher with horizontal use-wear marks. Retouch of blade end-scraper front, 4: retoucher oriented parallel to the front; 5: Blade end-scraper; 6: Retoucher with vertical use-wear marks.

dans son axe transversal et de celui du contour du support à retoucher. Ajoutons que l'orientation parallèle du retouchoir n'est qu'une solution parmi d'autres pour «gagner» de la surface active. Le tailleur peut aussi décider de retourner le fragment d'os et d'exploiter une zone offrant une morphologie plus favorable, voire de changer de retouchoir. Ainsi, l'orientation des retouchoirs expérimentaux n'a pas été systématiquement modifiée : à l'issue de la retouche totale d'une lame (bords et extrémité), les outils portent des plages composées d'impressions uniquement horizontales, verticales ou associant les deux types. Dans un cas comme dans l'autre, les stigmates sont généralement bien marqués (fig. 7 , $\mathrm{n}^{\text {os }} 3$ et 6$)$. Un retouchoir, toutefois, n'a conservé aucune trace de son utilisation mises à part quelques stries de glissement. Cette absence de stigmates, déjà constatée expérimentalement pour la retouche de racloirs (Armand et Delagnes, 1998, p. 212), est sans doute liée à la densité de l'os au niveau de sa zone d'exploitation.

\section{Le débitage de lamelles \\ à partir de nucléus carénés et à museau}

Plusieurs séries de lamelles ont été extraites à partir de nucléus carénés et à museau à l'aide de fragments d'os. Cependant, il est important de bien adapter le calibre des retouchoirs à celui des produits lithiques. Les hémi-côtes de bœuf n'ont effectivement pas permis de débiter les nucléus dont la table possède une longueur importante. Seuls l'ont permis les fragments diaphysaires, plus massifs. Précisons également que l'emploi des retouchoirs n'a pas inclus la préparation des nucléus. Cette opération nécessite en effet 
de débiter des éclats, ce que seul un percuteur plus lourd (dur ou tendre) autorise. L'emploi des retouchoirs expérimentaux s'est donc limité au détachement de lamelles (lamelles de mise en forme et de plein débitage). Les fragments diaphysaires de bœuf ont constitué des outils de taille appropriés et souples dans leur maniement. Les nucléus expérimentaux et les lamelles qui en ont été extraites entrent dans la gamme des produits rencontrés dans les ensembles archéologiques étudiés (fig. $9, n^{\text {os }} 3$ et 4). Notons

ORIENTATION DU RETOUCHOIR

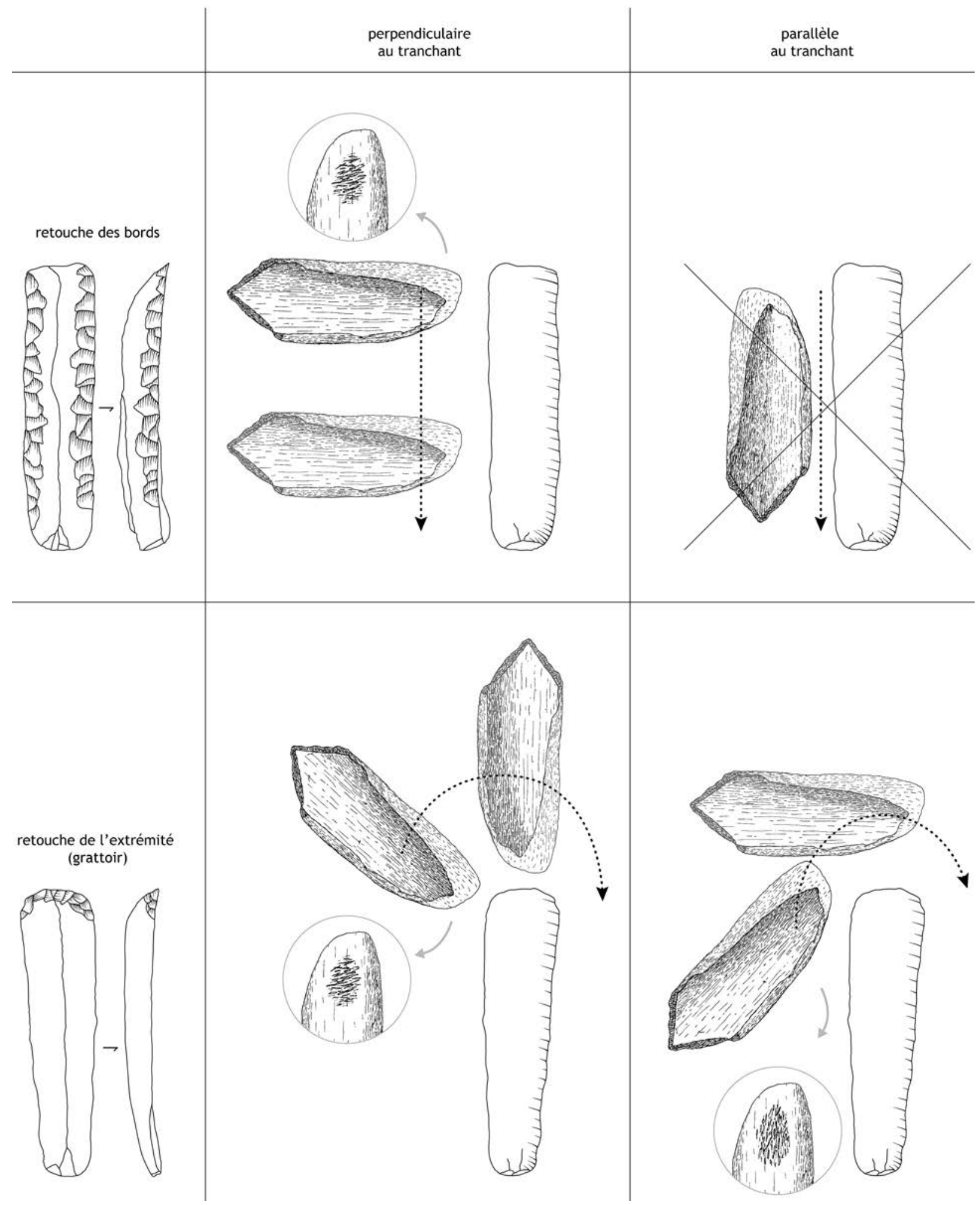

Fig. 8 - L'orientation des retouchoirs dans la retouche de lames (dessins : F. Le Mené, DAO : F. Tessier). Fig. 8-Orientation of retouchers during blade retouching (drawings: F. Le Mené, CAD: F. Tessier). 


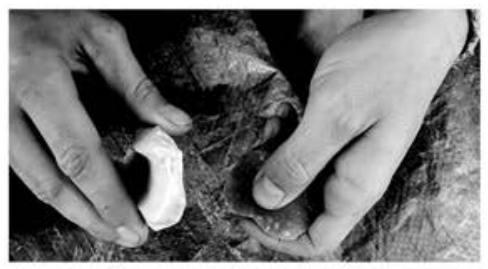

1

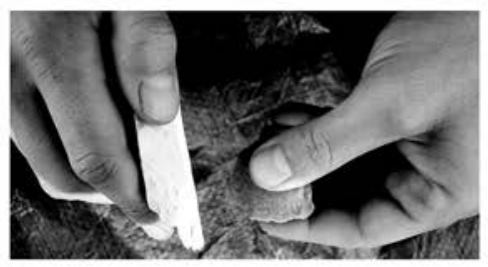

2

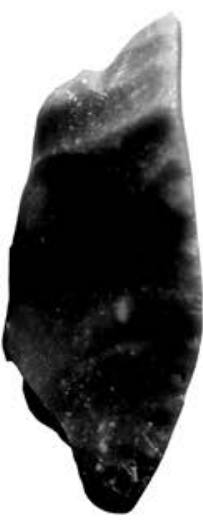

3
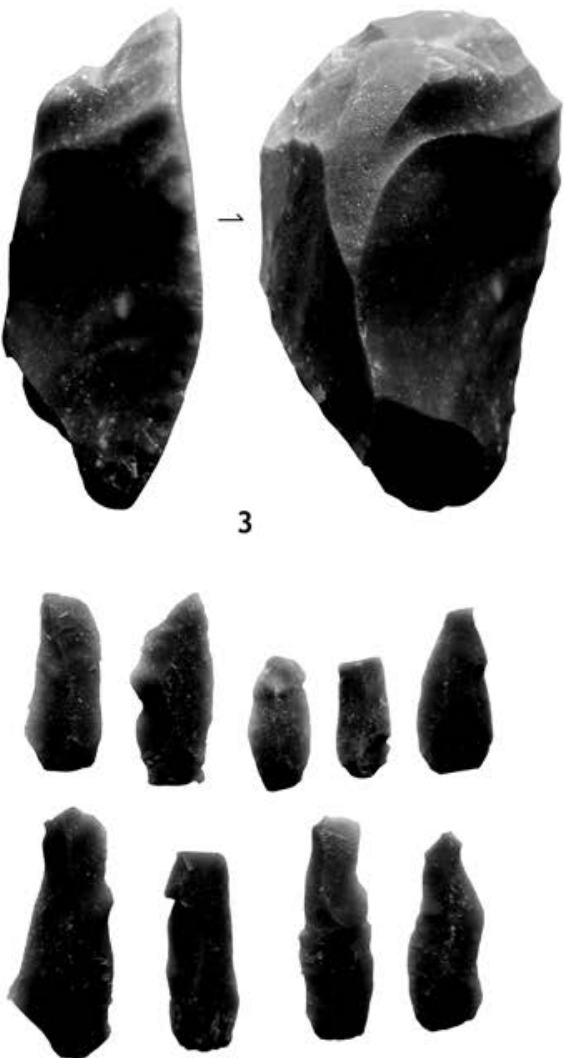

4

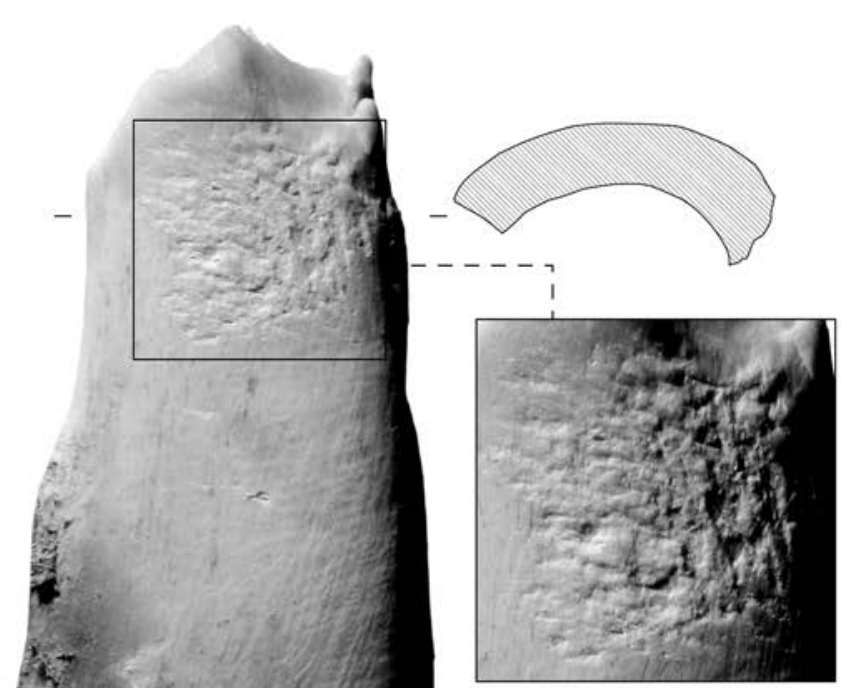

5

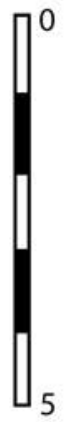

Fig. 9 - Utilisation expérimentale des retouchoirs pour le débitage laminaire, $1:$ retouchoir orienté perpendiculairement au bord de plan de frappe; 2 : retouchoir orienté parallèlement au bord de plan de frappe; $3:$ nucléus caréné à l'issue des tests; $4:$ exemples de lamelles débitées ; 5 : retouchoir à impressions mixtes : horizontales (impressions de gauche) et verticales (impressions de droite).

Fig. 9 - Experimental use of retouchers for bladelet production, 1: retoucher oriented perpendicular to the striking platform edge; 2: retoucher oriented parallel to the striking platform edge; 3: carinated core after the experiment; 4: examples of experimental bladelets; 5: retoucher with mixed use-wear marks: horizontal (to the left) and vertical (to the right).

toutefois qu'il a été assez difficile de contrôler la standardisation des lamelles. À ce stade des tests, cela ne préjuge pas nécessairement d'une efficacité moindre des outils osseux mais relève peut-être d'un manque de pratique des tailleurs, qui n'avaient encore jamais utilisé de retouchoir pour le débitage de lamelles.

Au même titre que pour la retouche des extrémités de lames, l'orientation parallèle du retouchoir s'est révélée efficace, la rectitude longitudinale de ce dernier s'adaptant bien à la délinéation convexe du bord de plan de frappe (fig. 10). Elle n'a cependant pas toujours été nécessaire, compte tenu de la faible courbure de certains retouchoirs dans l'axe transversal. À l'issue des tests, les stigmates présents sur les fragments d'os utilisés apparaissent assez proches de ceux observés sur les premières pièces expérimentales (fig. 9, $\mathrm{n}^{0} 5$ ). Les stigmates produits lors du débitage des lamelles semblent toutefois être plus profonds et souvent plus courts. 


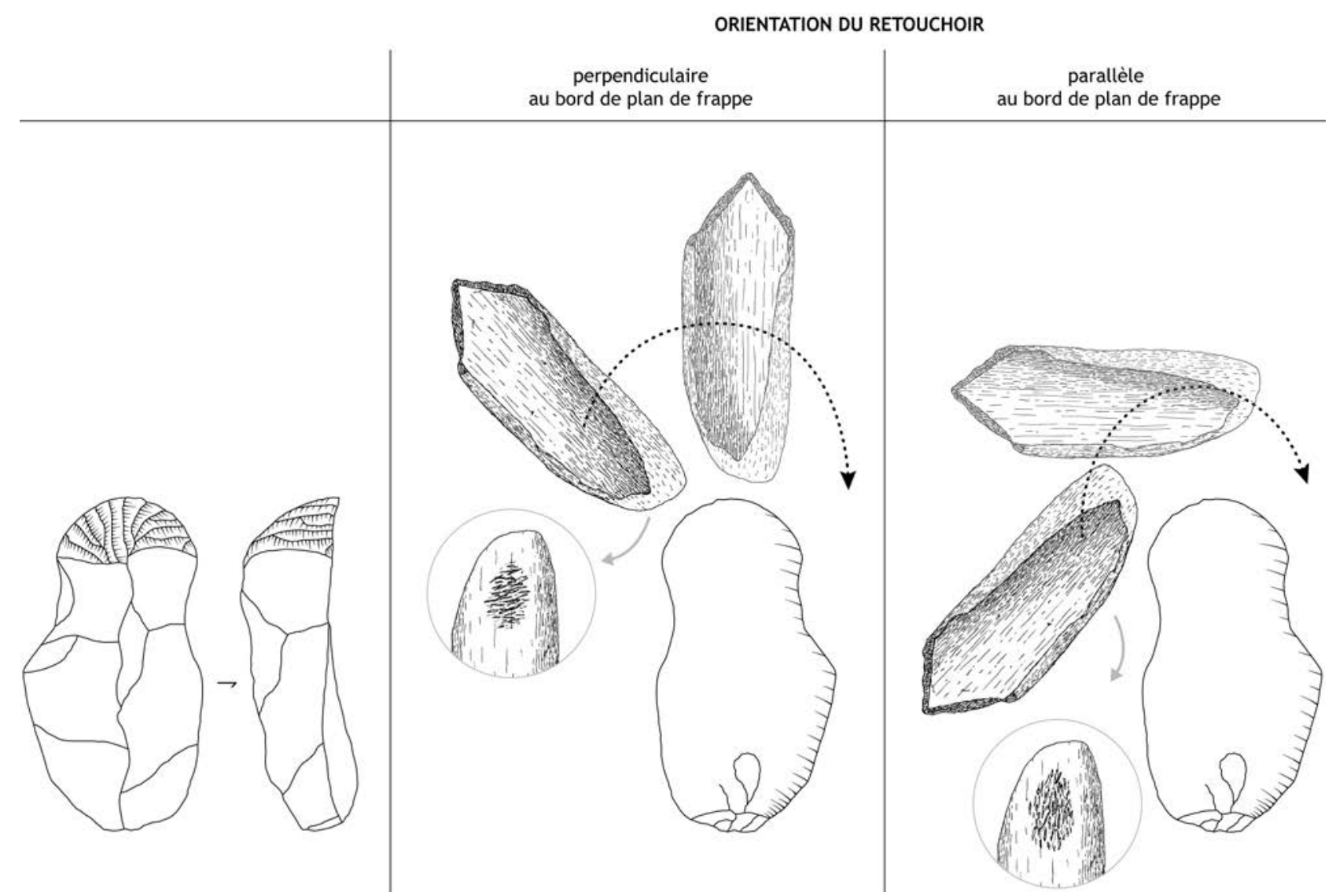

Fig. 10 - L'orientation des retouchoirs dans le débitage de lamelles (dessins : F. Le Mené, DAO : F. Tessier). Fig. 10-Orientation of retouchers during bladelet production (drawings: F. Le Mené, CAD: F. Tessier).

\section{RÉSULTATS EXPÉRIMENTAUX ET IMPLICATIONS ARCHÉOLOGIQUES}

Les tests réalisés ont montré que les retouchoirs en os constituent des outils efficaces pour la retouche de lames et permettent de débiter des lamelles à partir de nucléus carénés et à museau. Ils ont confirmé l'intérêt d'une orientation parallèle du retouchoir pour adapter la surface active aux zones de forte convexité des produits lithiques. Cela ne s'est pas révélé toujours nécessaire et dépend de la courbure transversale des retouchoirs ainsi que du degré de convexité des extrémités de lames et des bords de plan de frappe des nucléus. Par ailleurs, d'autres solutions existent pour gagner de la surface active, comme exploiter une zone du retouchoir plus favorable ou changer d'outil. Toujours est-il que la forte convexité des produits lithiques est à nos yeux le facteur le plus à même d'expliquer le changement d'orientation des retouchoirs au moins à compter de l'Aurignacien ancien. Ainsi, les retouchoirs de cette période attestent la perduration d'un procédé technique ancien, impliquant l'utilisation de l'os dans la retouche de l'outillage lithique. Les exemplaires porteurs d'impressions verticales témoigneraient d'une adaptation du fonctionnement des outils osseux aux nouveaux types de supports lithiques sur lesquels ils sont employés.
En réalité, le rôle important tenu par les retouchoirs dans la retouche de l'outillage au Paléolithique moyen et leur forte représentation au sein des ensembles étudiés laissaient peu de doutes concernant leur participation à la confection et à l'entretien de l'outillage sur lame à l'Aurignacien ancien. En revanche, il est encore difficile de déterminer s'ils ont véritablement joué un rôle dans le débitage des lamelles. Les tests ont montré l'efficacité des retouchoirs de gros calibre (os d'ongulé de grande taille type bovinés, cheval) qui sont présents en proportions variables dans les différentes séries étudiées (respectivement $50 \%$ et $28 \%$ des retouchoirs pour les secteurs nord et sud de Castanet, $76 \%$ à la grotte des Hyènes et $100 \%$ à Gatzarria). Mais les stigmates formés à la surface sont proches de ceux observés sur les retouchoirs expérimentaux utilisés dans la retouche de lames. S'ils semblent plus profonds et relativement plus courts, cela ne peut constituer, à ce stade de nos recherches, un critère suffisamment pertinent pour être transposé sur le plan archéologique. Un programme expérimental plus vaste couplé à une étude tracéologique à fort grossissement des outils osseux devrait permettre de mieux apprécier les variations morphométriques des stigmates en fonction de l'activité exercée. En complément, une comparaison des caractéristiques techniques et fonctionnelles ${ }^{8}$ des pièces lithiques archéologiques et expérimentales devrait aider à 
préciser le(s) type(s) de percuteur(s) utilisé(s) à l'Aurignacien ancien.

\section{DISCUSSION}

Les recherches consacrées à l'industrie en matières osseuses aurignacienne ont surtout mis l'accent sur les productions en bois de cervidé et en ivoire. L'équipement en os domine pourtant les séries en raison du nombre élevé d'outils bruts dont l'importance avait été sous-estimée. Les retouchoirs dont il est question ici en sont le meilleur exemple, mais ils ne constituent qu'une catégorie parmi un plus large registre d'outils non façonnés (Tartar, 2009). Cette réappréciation de la composition de l'industrie en matières osseuses de l'Aurignacien ancien appelle plusieurs remarques.

En premier lieu, l'importance de l'équipement en os implique d'élargir le champ d'application des recherches. De fait, l'exploitation des matières osseuses à l'Aurignacien ancien se caractérise par une nette partition fonctionnelle entre le bois de cervidé tourné vers la chasse, l'ivoire réservé à la parure et l'os destiné aux activités domestiques (Liolios, 2004; Tartar et al., 2006). Les sphères cynégétique et symbolique, à forte valeur sociale et empruntes d'un haut degré de technicité, ont jusque-là concentré l'essentiel des études. Il importe de se pencher également sur l'équipement en os, techniquement moins remarquable, mais dont la vocation domestique en fait un jalon indispensable à la reconstitution de la structuration socio-économique des groupes aurignaciens. Dans le cas des retouchoirs, il est question de l'organisation économique relative à la production des équipements lithiques et de l'interface des sous-systèmes techniques lithique et osseux. Il est encore trop tôt pour prendre la mesure exacte de l'implication des retouchoirs dans la production lithique. Si leur emploi dans la retouche des supports laminaires fait peu de doute, notre étude montre que leur participation au débitage lamellaire est une possibilité à ne pas négliger. Évoluant d'outils de retouche à celui de petits percuteurs, les retouchoirs pourraient avoir assumé une partie des fonctions jusque-là imputées uniquement au bois de cervidé et au bois végétal (ainsi qu'à la pierre tendre?), élargissant ainsi le registre des percuteurs tendres. L'étude fonctionnelle des retouchoirs de l'Aurignacien ancien ouvre ainsi de nouvelles pistes de réflexion pour discuter de la coexistence et du poids respectif des différentes techniques de percussion au sein des chaînes opératoires de productions lithiques.

En second lieu, cette réappréciation de la composition de l'industrie en matières osseuses de l'Aurignacien ancien implique que les changements technologiques marquant le passage entre le Paléolithique moyen et supérieur ne sont pas aussi francs que ce qu'on a pu envisager. Longtemps décrit sous l'angle de la rupture, les données livrées par l'industrie lithique suggèrent que cet épisode s'inscrirait au contraire dans une évolution technologique et conceptuelle lente et graduelle (Teyssandier et al., 2010). C'est aussi ce qu'évoque l'industrie en matières osseuses. Si l'exploitation du bois de cervidé et en particulier la production d'éléments d'armature marquent un cap et sont des éléments forts caractérisant l'Aurignacien et plus largement le Paléolithique supérieur en Europe, les témoignages restent rares avant la phase ancienne, au Protoaurignacien ou Aurignacien archaïque (Teyssandier et Liolios, 2008). Qui plus est, sauf dans quelques sites exceptionnellement riches (Castanet, Isturitz, etc.), l'exploitation du bois de cervidé comme celle de l'ivoire sont encore relativement peu développées à l'Aurignacien ancien. Dorénavant, il faut compter également sur un outillage en os non façonné bien plus abondant que supposé, dont certains éléments (les retouchoirs parmi d'autres) composaient déjà les ensembles osseux moustériens.

Remerciements : Mes premiers remerciements reviennent à Marianne Christensen et F. Le Mené pour leurs remarques et suggestions lors de la rédaction de cet article ainsi qu'à Claire Heckel pour ses corrections des textes en anglais. J'adresse aussi mes remerciements à Dominique Henry-Gambier, François Lévêque (alors légataire testamentaire de Georges Laplace), Jacques Pelegrin et Randall White pour m'avoir confiée pour étude les produits de leurs fouilles. Je remercie également les conservateurs et personnels des musées qui m'ont accueillie et facilitée l'accès aux collections : à Catherine Schwab et Marie-Sylvie Larguèze du musée d'Archéologie nationale de Saint-Germain-enLaye; à Jean-Jacques Cleyet-Merle, André Morala, Peggy Jacquement et Bernard Nicolas du musée national de Préhistoire des Eyzies.

\section{NOTES}

(1) La série comprend les pièces isolées à la fouille et celles retrouvées postérieurement, parmi les restes osseux récupérés au sein des déblais.

(2) Publications en préparation : J. Pelegrin dir., L'abri Castanet, Sergeac, Dordogne. Fouilles du nouveau secteur (1994-1998), numéro spécial de Paléo et R. White, R. Bourrillon, A. Clark, L Chiotti., C. Cretin, R. Mensan, A. Morala, M. Sisk et É. Tartar, L'Aurignacien du vallon de Castel-Merle (commune de Sergeac, Dordogne) à la lumière des fouilles récentes de l'abri Castanet (2005-2010).

(3) Le corpus de pièces pour le secteur sud correspond aux exemplaires récoltés entre 1995 et 2007.

(4) Publication en préparation : D. Henry-Gambier et F. Bon dir., L'Aurignacien de la grotte des Hyènes (Brassempouy, Landes).

(5) Dans l'Aurignacien archaïque d'Isturitz, couche C4d1 (Soulier et Goutas, à paraître) ainsi que, semble-t-il, dans des niveaux plus récents: dans la couche 4 de La Rochette (obs. pers.), rapportée à l'Aurignacien II (Delporte, 1962); dans la couche B des Rois (Mouton et Joffroy, 1958), attribuée probablement à un Aurignacien moyen récent (D'Errico et al., 2010). À noter également un retouchoir sur canine inférieure (défense) de sanglier provenant de la couche K, protoaurignacienne, du Piage (fouilles Champagne et Espitalié : Tartar, 2006).

(6) Les impressions horizontales (ou légèrement obliques) forment un angle compris entre 46 et $90^{\circ}$ avec l'axe longitudinal des outils et les impressions verticales (ou légèrement obliques) un angle compris entre 0 et $45^{\circ}$.

(7) Les tests réalisés avec M. Brenet ont intégré un programme expérimental plus vaste dont l'objectif était de définir des critères de reconnaissance pour les micro-vestiges lithiques retrouvés en contexte archéologique. Il s'est déroulé à l'Abri Castanet (Sergeac, Dordogne), lors de la campagne de fouille 2006, avec la collaboration de L. Chiotti, A. Clark et C. Cretin.

(8) Une étude tracéologique des lamelles et des nucléus à lamelles de l'abri Castanet (secteur sud) par Maureen Hays est déjà programmée. 


\section{RÉFÉRENCES BIBLIOGRAPHIQUES}

ARMAND D., DELAGNES A. (1998) - Les retouchoirs en os d'Artenac (couche 6c) : perspectives archéozoologiques, taphonomiques et expérimentales, in J.-P. Brugal, L. Meignen et M. Patou-Mathis dir., Économie préhistorique : les comportements de subsistance au Paléolithique, actes des $18^{\text {es }}$ Rencontres internationales d'archéologie et d'histoire d'Antibes, Antibes, 1997, Sofia Antipolis, APDCA, p. 205-214.

CASTEL J.-C., MADELEINE S. (2003) - Stigmates observés sur les dents de grands carnivores à l'Aurignacien. L'exemple de l'abri de la Souquette à Sergeac (Dordogne, France), Paléo, 15, p. 251-254.

CASTEL J.-C., CHAUVIÈRE F.-X. et MADELEINE S. (2003) - Sur os et sur dents : Les «retouchoirs» aurignaciens de La Ferrassie (Savignac-de-Miremont, Dordogne), Paléo, 15, p. 29-50.

CHASE P. G. (1990) - Tool-making Tools and Middle Paleolithic Behavior, Current Anthropology, 31, p. 443-447.

CHIOTTI L. (2000) - Lamelles Dufour et grattoirs aurignaciens (carénés et à museau) de la couche 8 de l'abri Pataud, Les Eyzies-de-Tayac, Dordogne, L'Anthropologie, 104, p. 239-263.

DALEAU F. (1883) - Sur des lésions que présentent certains os de la période paléolithique, Congrès de l'Association française pour l'avancement des Sciences, $12^{\mathrm{e}}$ session, Rouen, Paris, [s. n.], p. 600-602.

DELPORTE H. (1962) - Le gisement paléolithique de La Rochette (Dordogne), Gallia Préhistoire, 5, 1, p. 1-22.

DIDON L. (1911) - L'Abri Blanchard des Roches, Bulletin de la Société historique et archéologique du Périgord, 87, p. 246-241 et 321-345.

D'ERRICO F., MARIAN VANHAEREN M., RENOU S., MICHEL A., LENOBLE A., VERNA C., RAMIREZ ROZZI F., RENDU W., ROGER G. (2010) - Nouvelles fouilles dans le site aurignacien de Chez les Rois (Mouthiers-sur-Boëme, Charente), in J. Buisson-Cattil et J. Primault dir., Préhistoire entre Vienne et Charente. Hommes et sociétés du Paléolithique, 25 ans d'archéologie préhistorique en Poitou-Charentes, Chauvigny, Association des publications chauvinoises, ministère de la Culture et de la Communication (Mémoire 38), p. 245-253.

GIRAUX L. (1907) - Ossements utilisés de Cro-Magnon, Bulletin de la Société préhistorique française, 4, 5, p. 264-268.

HAHN J. (1970) - Recherches sur l'Aurignacien en Europe centrale et orientale, L'Anthropologie, 74, 3-4, p. 195-220.

HENRI-MARTIN L. (1907-1910) - Recherches sur l'évolution du Moustérien dans le gisement de La Ouina (Charente). L'industrie osseuse, Paris, Schleicher, $315 \mathrm{p}$.

HENRY-GAMBIER D., BON F., GARDERE P., LETOURNEUX C., MENSAN R., POTIN Y. (2004) - Nouvelles données sur la séquence culturelle du site de Brassempouy (Landes) : fouilles 1997-2002, Archéologie des Pyrénées occidentales et des Landes, 23, p. 145-156.

KARAVANIC I., SOKEC T. (2003) - The Middle Paleolithic Percussion or Pressure Flaking Tools? The Comparison of Experimental and Archaeological Material from Croatia, Prilozi Instituta za Arheoogiju u Zagrebu, 20, p. 5-14.

LAPLACE G. (1966) - Les niveaux castelperroniens, protoaurignaciens et aurignaciens de la grotte Gatzarria à Suhare en Pays Basque (fouilles 1961-1963), Quartär, 17, p. 117-139.

LE BRUN-RICALENS F. (2005) - Chronique d'une reconnaissance attendue. Outils «carénés», outils «nucléiformes»: nucléus à lamelles. Bilan après un siècle de recherches typologiques, technologiques et tracéologiques, in F. Le Brun-Ricalens dir., avec la collab. de J.-G. Bordes et F. Bon, Productions lamellaires attribuées à l'Aurignacien : chaînes opératoires et perspectives techno-culturelles, actes de la table ronde organisée dans le cadre du XIV Congrès de l'UISPP, Liège, 2001, Luxembourg, Musée national d'histoire et d'art (Archéologiques 1), p. 23-72.

LEGUAY L. (1877) - Les procédés employés pour la gravure et la sculpture des os avec les silex, Bulletin de la Société d'anthropologie de Paris, $2^{\mathrm{e}}$ série, 12, p. 280-296.
LEROY-PROST C. (1975) - L'industrie osseuse aurignacienne. Essai régional de classification : Poitou, Charente, Périgord, Gallia Préhistoire, 18, 1, p. 65-156.

LEROY-PROST C. (2002) - Objets sur meule de bois de cervidés, in M. Patou-Mathis dir., Fiches typologiques de l'industrie osseuse préhistorique. Commission de nomenclature sur l'industrie de l'os préhistorique. Cahier X: Os à impressions et éraillures, Treignes, CEDARC, p. 105-115.

LIOLIOS D. (2004) - Le travail des matières osseuses au début de l'Aurignacien : aspects techniques, économiques et symboliques de l'organisation de la production de Geißenklösterle (Jura souabe), in P. Bodu et C. Constantin dir., Approches fonctionnelles en Préhistoire, XXV Congrès préhistorique de France, Nanterre, 2000, Paris, Société préhistorique française et ministère de la Culture, p. 371-386.

MALERBA G. et GIACOBINI G. (1996) - Les retouchoirs sur éclats diaphysaires du Paléolithique moyen et supérieur de trois sites de l'Italie nord orientale (grotte de San Bernardino, abri de Fumane et abri Tagliente), in M. Patou-Mathis éd., Workshop 4. Lower and Middle Palaeolithic Bone Industries : New Analytical Methods, actes du XIII ${ }^{\mathrm{e}}$ Congrès de l'UISPP, Forli, ABACO, p. 167-172.

MOUTON P., JOFFROY R. (1958) - Le gisement aurignacien des Rois à Mouthiers (Charente), Paris, CNRS (Supplément à Gallia Préhistoire, $9,140 \mathrm{p}$.

PATOU-MATHIS M. (2002) - Fiches typologiques de l'industrie osseuse préhistorique. Commission de nomenclature sur l'industrie de l'os préhistorique. Cahier X: Os à impressions et éraillures, Treignes, CEDARC, $136 \mathrm{p}$

PEYRONY D. (1935) - Le gisement de Castanet, vallon de Castelmerle, commune de Sergeac (Dordogne), Bulletin de la Société préhistorique française, 32,9 , p. 418-443.

RIGAUD A. (1977) - Analyses typologique et technologique des grattoirs magdaléniens de La Garenne à Saint-Marcel (Indre), Gallia Préhistoire, 20, p. 1-43.

RIGAUD A. (2007) - Retouchoirs sur éclats diaphysaires ou «affûtoirs » de Labastide (Hautes-Pyrénées). Du Barbarisme scientifique à la rigueur artisanale au travers de l'expérimentation, Archéologie des Pyrénées occidentales et des Landes, 26, p. 193-200.

SCHWAB C. (2002) - Éclats diaphysaires du Paléolithique moyen et supérieur : la grotte d'Isturitz (Pyrénées-Atlantiques), in M. PatouMathis dir., Fiches typologiques de l'industrie osseuse préhistorique. Commission de nomenclature sur l'industrie de l'os préhistorique. Cahier X : Os à impressions et éraillures, Treignes, CEDARC, p. 59 74.

SCHWAB C. (2009) - Les « os à impressions et à éraillures» : premiers résultats expérimentaux, Antiquités nationales, 40, p. 29-37.

SOULIER M.-C., GOUTAS N. (à paraître) - L'exploitation des ressources animales dans l'Aurignacien archaïque de la grotte d'Isturitz (Pyrénées-Atlantiques, France) : regards croisés de l'archéozoologue et du technologue, in $\mathrm{C}$. Thiébault, E. Claud et S. Costamagno dir., Exploitation des ressources organiques à la fin du Paléolithique moyen et au début du Paléolithique supérieur : interactions entre environnement et comportements techniques, actes de la session E du XXVII Congrès préhistorique de France, Bordeaux, 2010, Paris, Société préhistorique française, à paraître.

TARTAR É. (2006) - L'industrie en matières dures animales du Piage, in J.-G. Bordes, F. Lebrun-Ricalens et collab., Rapport de fouille programmée du Piage (Fajoles, Lot), campagne 2006, 10 p.

TARTAR É. (2009) - De l'os à l'outil : caractérisation technique, économique et sociale de l'utilisation de l'os à l'Aurignacien ancien. Étude de trois sites : l'Abri Castanet (secteurs nord et sud), Brassempouy (grotte des Hyènes et abri Dubalen) et Gatzarria, thèse de doctorat, université Paris I - Panthéon-Sorbonne, 2 vol., 300 p.

TARTAR É. (à paraître) - Le travail de l'os à l'Aurignacien ancien réflexion pour une interprétation techno-économique de l'outillage à partir du matériel de la grotte des Hyènes et de l'abri Dubalen, in 
D. Henry-Gambier et F. Bon (dir.), L'Aurignacien de la grotte des Hyènes (Brassempouy, Landes), à paraître.

TARTAR É, TEYSSANDIER N., BON F., LIOLIOS D. (2006), Équipement de chasse, équipement domestique : une distinction efficace? Réflexion sur la notion d'investissement technique dans les industries aurignaciennes, in L. Astruc, F. Bon, V. Lea, P.-Y. Milcent et S. Phillibert dir., Normes techniques et pratiques sociales : de la simplicité des outillages pré- et protohistoriques, Antibes, ADPCA p. 107118.

TEYSSANDIER N., BORDES J.-G., BON F. (2010) - Within Projectile Range. Some Thoughts on the Appearance of the Aurignacian in Europe, Journal of Anthropological Research, 66, 2, p. 209-229.

TEYSSANDIER N., LIOLIOS D. (2008) - Le concept aurignacien : entre rupture préhistorique et obstacle épistémologique. Bulletin de la Société préhistorique française, 105, 4, p. 737-747.
VÉZIAN J., VÉZIAN J. (1970) - Les gisements de la grotte de SaintJean-de-Verges, Ariège, Bulletin de la Société préhistorique de l'Ariège, 25, p. 29-77.

VINCENT A. (1993) - L'outillage osseux au Paléolithique moyen : une nouvelle approche, thèse de doctorat, université Paris X - Nanterre, 2 vol., $331 \mathrm{p}$

\section{Élise TARTAR}

UMR 7041 «Ethnologie préhistorique» Maison de l'archéologie et de l'ethnologie 21, allée de l'Université, 92023 Nanterre cedex elise.tartar@gmail.com 